\title{
Saccadic flight strategy facilitates collision avoidance: closed-loop performance of a cyberfly
}

\author{
Jens Peter Lindemann • Holger Weiss • Ralf Möller • \\ Martin Egelhaaf
}

Received: 18 September 2006 / Accepted: 29 November 2007 / Published online: 8 January 2008

(C) Springer-Verlag 2008

\begin{abstract}
Behavioural and electrophysiological experiments suggest that blowflies employ an active saccadic strategy of flight and gaze control to separate the rotational from the translational optic flow components. As a consequence, this allows motion sensitive neurons to encode during translatory intersaccadic phases of locomotion information about the spatial layout of the environment. So far, it has not been clear whether and how a motor controller could decode the responses of these neurons to prevent a blowfly from colliding with obstacles. Here we propose a simple model of the blowfly visual course control system, named cyberfly, and investigate its performance and limitations. The sensory input module of the cyberfly emulates a pair of output neurons subserving the two eyes of the blowfly visual motion pathway. We analyse two sensory-motor interfaces (SMI). An SMI coupling the differential signal of the sensory neurons proportionally to the yaw rotation fails to avoid obstacles. A more plausible SMI is based on a saccadic controller. Even with sideward drift after saccades as is characteristic of real blowflies, the cyberfly is able to successfully avoid collisions with obstacles. The relative distance information contained in the optic flow during translatory movements between saccades is provided to the SMI by the responses of the visual output neurons. An obvious limitation of this simple mechanism is its strong dependence on the textural properties of the environment.
\end{abstract}

J. P. Lindemann $(\varangle) \cdot$ H. Weiss $\cdot$ M. Egelhaaf

Neurobiologie, Fakultät für Biologie, Universität Bielefeld,

Postfach 1001 31, 33501 Bielefeld, Germany

e-mail: Jens.Lindemann@Uni-Bielefeld.DE

R. Möller

AG Technische Informatik, Technische Fakultät,

Universität Bielefeld, Postfach 1001 31, 33501 Bielefeld, Germany
Keywords Motion vision - Behaviour - Model simulation . Saccadic flight

\section{Introduction}

Anyone who has observed a blowfly landing on the rim of a cup or two blowflies chasing each other will be familiar with the breath-taking aerobatics these tiny animals can produce. Within a fraction of a second the blowfly performs several high-speed saccadic turns in preparation for a safe landing, and a pursuer blowfly is quite capable of catching its speeding target. The human eye is scarcely able to follow the flight paths of flies performing such behaviours. In contrast to this, blowflies skilfully avoid obstacles emerging in their flight path during these high speed flight manoeuvres. Obstacle detection relies largely on the continually changing flow of the retinal images of the environment. This optic flow is delivered to the brain, evaluated within $20-30 \mathrm{~ms}$ (Warzecha and Egelhaaf 2000) and transformed into signals the blowfly uses for motor control (Egelhaaf 2006). The correctional movements, in turn, result in changes of the retinal image flow, closing the action-perception loop.

The blowfly has proved to be an outstanding model system for unravelling the computations in the brain which serve to extract the behaviourally relevant information from the retinal optic flow patterns (Borst and Haag 2002; Egelhaaf 2006; Hausen 1993; Krapp 2000). On the one hand, large parts of the blowfly visual system are involved in optic flow processing and, on the other hand, experimental analysis can be performed on blowflies by a broad spectrum of methods. Only recently it became possible, thanks to novel technologies, to investigate optic flow processing using visual stimuli which come very close to what flies have seen during their acrobatic flight manoeuvres (Boeddeker et al. 2005; 
Karmeier et al. 2006; Kern et al. 2005; Lindemann et al. 2003; van Hateren et al. 2005).

Based on experimental results, motion computation in the blowfly visual motion pathway has been modelled to various degrees of sophistication (Borst et al. 2003; Borst 2004; Kern et al. 2000, 2001), culminating in the finding that these models can even account for the performance of an output neuron of the blowfly motion vision system under naturalistic stimulus conditions (Lindemann et al. 2005). So far, however, all these models focus on the mechanisms underlying sensory information processing and have only rarely been embedded as the sensory input stage in a more comprehensive model of blowfly visuo-motor control (Warzecha and Egelhaaf 1996).

Although blowflies were observed to be able to fly slow continuous turns while chasing targets (Boeddeker et al. 2003; Boeddeker and Egelhaaf 2003), they do not show this behaviour in normal cruising flight or in obstacle avoidance tasks. Instead, they keep their gaze almost perfectly stable for short flight segments and then execute sharp fast turns, commonly referred to as body saccades. These saccades only last for about 50-100 ms. Since during saccades blowflies may reach rotational velocities of up to $5,000^{\circ} / \mathrm{s}$, they can change their body orientation by up to $90^{\circ}$ in this short time (Schilstra and van Hateren 1999; van Hateren and Schilstra 1999). During saccadic turns, the head and body movements are coordinated in a way that minimises the time period of unstable gaze. Head movements serve to temporally shorten the turn with respect to the gaze. This behaviour can be interpreted as an active vision strategy stabilising the gaze rotationally as much as possible (Kern et al. 2006; Schilstra and van Hateren 1998). By this gaze stabilisation, the rotational and translational components of the optic flow are largely segregated at the behavioural level. However, in blowflies the saccadic strategy of flight and gaze control is not obviously reflected as sharp bends in the path of the body's centre of mass. Changes in orientation do not immediately result in changes in flight direction due to sideward drift after a body saccade, presumably caused by the inertia of the blowfly (Schilstra and van Hateren 1999).

The separation of rotational and translational optic flow components is assumed to facilitate the extraction of spatial information from the optic flow, because only the translational flow component provides information about the distance to environmental objects (Koenderink and van Doorn 1987). Pooling of optic flow information over a large part of the visual field into the activity of a single neuron drastically reduces the amount of information to be processed by subsequent stages of the system. Nonetheless, the relevant information may still be encoded in the integrated signal, if the spatial pooling is done appropriately (Dahmen et al. 1997). Indeed, motion sensitive output neurons of the blowfly's visual motion pathway, such as the HS-cells, are supposed to extract behaviourally relevant information about translational optic flow. In the intersaccadic intervals of relatively stable gaze they encode information about the threedimensional structure of the environment (Kern et al. 2005; van Hateren et al. 2005). Nonetheless, it is not clear so far what triggers saccades in blowflies. For the fruit fly Drosophila, it was concluded that a visual trigger feature for saccades is fronto-lateral image expansion (Bender and Dickinson 2006; Tammero and Dickinson 2002a,b).

Given the ability of flies to perform extraordinarily acrobatic flight manoeuvres, it may not be surprising that there have been various attempts to implement fly-inspired optic flow models in simulation and on robotic platforms (Franz et al. 2004; review: Franz and Mallot 2000; Neumann 2004; Webb et al. 2004; Zufferey and Floreano 2006). On the one hand, these approaches usually employed simplified versions of the sensory models. On the other hand, they did not attempt to model the characteristics of fly visually guided behaviour. However, in all these studies the sensory-motor loop was closed. Most of these systems use optic flow information to stabilise the path of locomotion against disturbances. There are only few attempts in robotics to make use of the saccadic strategy of locomotion and of the implicit distance information present in translatory optic flow to implement obstacle avoidance (Chahl and Srinivasan 2000; Franceschini et al. 1992; Reiser and Dickinson 2003; Sobey 1994; Zufferey and Floreano 2006). However, most of these systems generate movements of very slow dynamics compared to the blowfly.

The system described in the following uses a saccadic controller that receives its sensory input from a model of the fly's visual motion pathway and takes the specific dynamic features of blowfly behaviour into account. The sensory model providing the input to the controller has been specified and parameterised on the basis of experimental data (Lindemann et al. 2005). The calibration was based on responses of the HSE-cells, major motion sensitive output neurons of the blowfly's visual system (Hausen 1982a,b), to naturalistic optic flow, i.e., the visual input of flies in free-flight situations (Kern et al. 2005). To simulate the behaviour of a blowfly, the output signals of the sensory model must be transformed into motor signals to generate behavioural responses. The properties of the motor controller determine how these motor signals are transformed into movements of the animal. By simulating the system in a closed control loop, hypotheses about the functional significance of the responses of sensory neurons and different types of SMIs will be tested in the context of obstacle avoidance. Here we study the ability of the controller to avoid collisions with obstacles, one of the most fundamental tasks of any autonomous agent. The optomotor response was proposed in a previous study to be able to prevent flies from colliding with the walls of an experimental arena (Götz 1975). To test this hypothesis, the performance of a smooth optomotor controller will be compared with that of a saccadic controller. The main purpose of this study is 


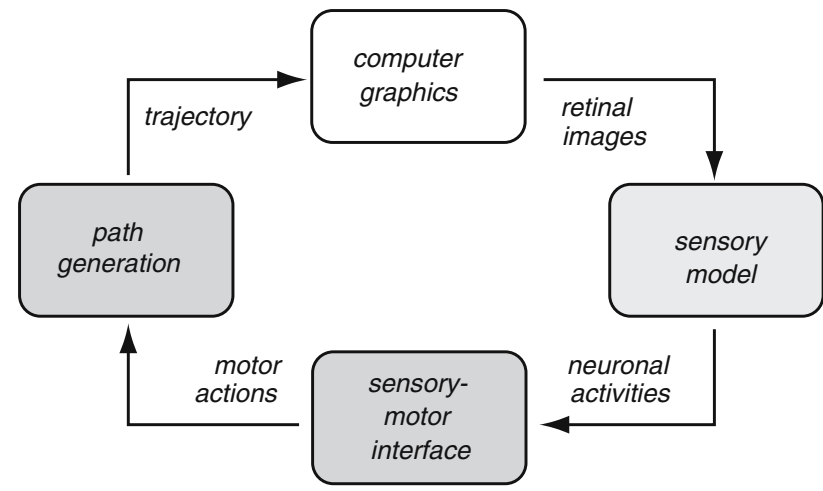

Fig. 1 Principle of the closed-loop simulation. The retinal images generated in a computer graphics module are fed to the model of the sensory system. The responses of the sensory system are converted by the sensory-motor coupling module to motor actions. The path generation module generates an update of the cyberfly's position and orientation from these. The loop is closed by generating the next retinal image frame for this updated position

to test hypothetical mechanisms for visually guided obstacle avoidance behaviour of blowflies. However, the developed flight controllers may also serve as part of biologically inspired autonomous artificial systems. Simple and efficient mechanisms for autonomous course control and, in particular, the avoidance of obstacles are of crucial significance in the technical domain.

\section{The model}

\subsection{Closed-loop simulation environment}

Closed-loop simulations can be used to test hypotheses regarding the coupling of the sensory system responses to the behavioural output. In our implementation, a computer graphics system generates a retinal image, based on the position and orientation of the cyberfly in a predefined virtual 3D-environment (Fig. 1). The retinal images are processed by a model of the sensory system that is then coupled to a SMI module implementing the formalised hypothesis for behavioural control. A simple path generation module calculates the movements of the virtual animal resulting from the signals generated by the SMI and updates the system's position and orientation in the virtual environment. The path generation phenomenologically takes into account the body dynamics of the fly. To close the loop, the new position is passed to the computer graphics system for the generation of a new retinal image.

\subsubsection{Sensory model}

The sensory model used for the closed-loop simulations corresponds to the model of one output neuron, the HSE-cell, in each half of the blowfly visual motion pathway. The HSE neuron responds to horizontal wide-field motion in the equatorial part of the visual field with graded membrane potential changes superimposed by small active potentials (Hausen 1982a,b). This model has previously been shown to account for all major features of the graded responses of the real cell to behaviourally generated optic flow (Lindemann et al. 2005). The sensory model is sketched here only briefly to make this paper self-contained (Fig. 2).

The input images of the visual motion pathway are sampled by Gaussian shaped spatial low-pass filters $\left(\sigma=2^{\circ}\right)$. The output of these filters forms the input to the photoreceptors that are equally spaced at $2^{\circ}$ along the elevation and azimuth of the eye. The array of photoreceptors forms a rectangular grid in the cylindrical projection with 51 rows and 86 columns. The temporal properties of the peripheral visual system are modelled as a temporal filter with a kernel that was derived from an electrophysiological analysis of the responses of 2nd-order visual interneurons (LMCs) with white-noise brightness fluctuations (James 1990; Juusola et al. 1995). The filter kernel is a kind of temporal band-pass filter and is shown in Fig. 2a (for a formal description see Lindemann et al. 2005).

The filter outputs of neighbouring elements are fed into elaborated movement detectors of the correlation type with a 1st-order temporal low-pass filter (time constant $10 \mathrm{~ms}$ ) in one of its branches and a 1st-order temporal high-pass filter (time constant $60 \mathrm{~ms}$ ) in its other branch (Fig. 2a). Each local movement detector consists of two mirror-symmetrical subunits. In each subunit, the low-pass filtered signal of one input channel is multiplied with the high-pass filtered signal of the neighbouring input channel. Before the outputs of the movement detector subunits are spatially pooled by the model HSE-cell, their signals are differently weighted, according to the spatial sensitivity distribution of the HSE-cell (Krapp et al. 2001). Maximal sensitivity of the simulated neuron is at $15^{\circ}$ lateral to the body long axis (see Lindemann et al. 2005 for details). The spatial pooling site of the model represents the dendritic tree of the HSE-neuron. The summation of the two types of motion detector subunits are interpreted as the conductances of excitatory and inhibitory synapses on the integrating cell (Borst et al. 1995). The resulting membrane potential of the model HSE-cell is calculated on the basis of the equivalent electrical circuit of a one-compartment passive membrane patch (Fig. 2a; for details see Lindemann et al. 2005). Since conductances cannot assume negative values, the outputs of the movement detector subunits were rectified before feeding them into the model of the integrating cell (Fig. 2a):

$V_{m}=\frac{E_{e} g_{e}+E_{i} g_{i}+E_{0} g_{0}}{g_{e}+g_{i}+g_{0}}$ 

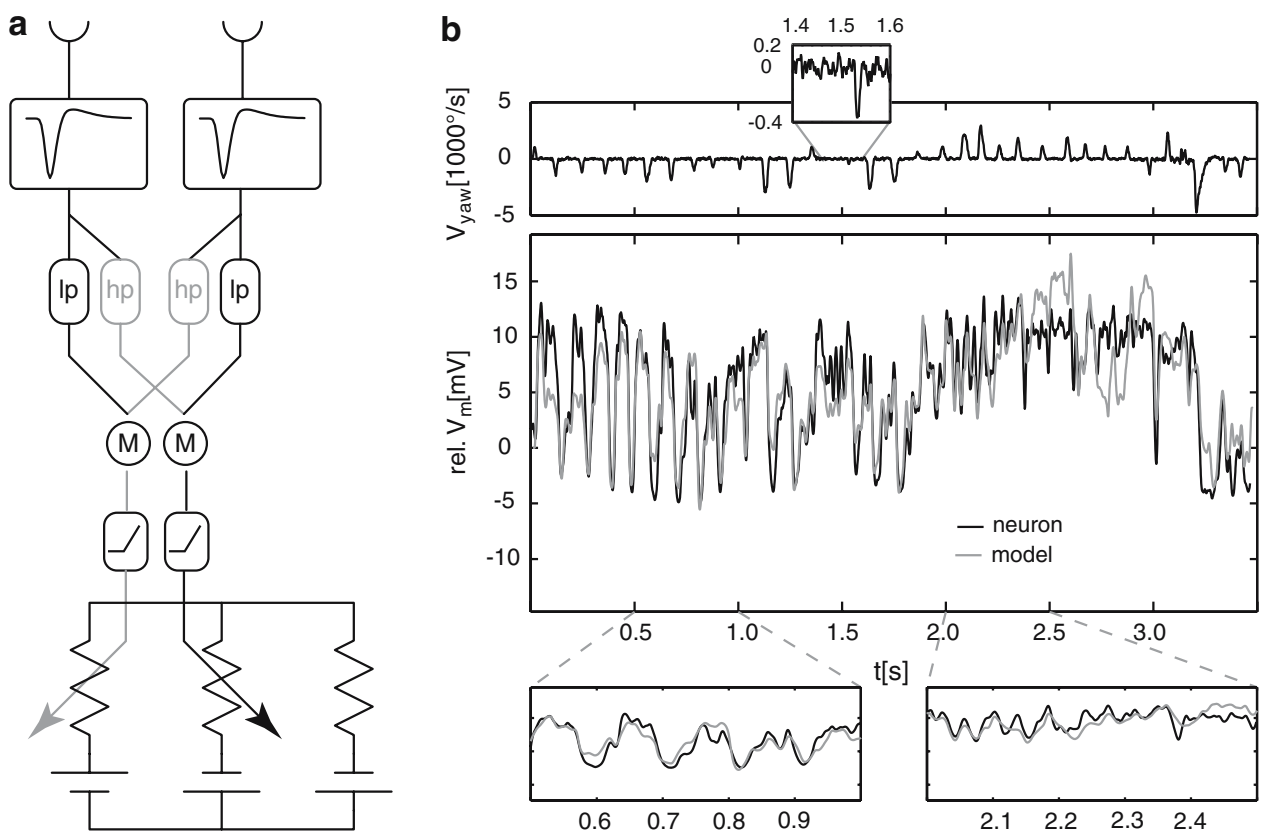

Fig. 2 a Structure of the sensory model. The signals of the input elements (Gaussian spatial lowpass filters not shown) are processed by a temporal convolution filter approximating the response of the 2nd-order interneurons in the fly visual system. These signals are fed to elaborated correlation-type motion detectors. The motion is computed from a pair of input elements by correlating (multiplying) the high-pass filtered signal of one to the low-pass filtered signal of a neighbouring channel. Two mirror-symmetrical subunits of this structure exist for each pair of input elements. The signals of these motion detector subunits

For further simplification of Eq. 1, the resting potential of the model HSE-cell was set to $E_{0}=0 \mathrm{mV}$. This setting does not change the relative fluctuations of the membrane potential. The excitatory $\left(g_{e}\right)$ and inhibitory $\left(g_{i}\right)$ synaptic conductances are given by the summed outputs of the two types of motion detector subunits after weighting them according to the spatial sensitivity distribution of the HSE-cell (see above). The leak conductance $g_{0}$ and the ratio of the inhibitory and excitatory synaptic reversal potentials $E_{i} / E_{e}$ are free parameters of the model. These two parameters were determined by optimisation of the quality of the fit of cellular responses and chosen as $g_{0}=500$ and $E_{i} / E_{e}=-0.95$, respectively.

With these parameter settings, the model of the HSE-cell was shown to fit the complex time course of the responses of its natural counterpart to behaviourally generated optic flow with a deviation comparable to the neuronal intertrial variability (Fig. 2b). In particular, the ability of the neuron to represent information about the optic flow generated by sideward movements was found in the model responses, too. This means that the model codes relative information about the three-dimensional layout of the environment between saccades like the neuron does (Lindemann et al. 2005). are spatially pooled by a weighted sum and drive the synaptic conductances of an excitatory and an inhibitory synapse in a one-compartment membrane model. b The response of this model to behaviourally generated optic flow closely fits the response of the real neuron to the same stimulus. The upper panel shows the yaw rotations present in the stimulus; the central panel shows the responses of the model (grey line) and the neuron (black line). The lower panels show example segments zoomed for closer inspection. Figure adapted from Lindemann et al. (2005)

\subsubsection{Sensory-motor interfaces (SMIs)}

Two different modules for the coupling of the sensory neurons to the motor output were tested in closed loop simulation with respect to obstacle avoidance: A simple continuous coupling of the large-field motion sensitive neurons to the rotation implementing the so-called optomotor response, and a controller generating saccadic turns as observed in free flying blowflies. A detailed description of the two SMIs is given below alongside with the results obtained with the particular module.

Although, in principle, it is possible to estimate the flight forces resulting from the induced wing beat pattern (Schenato et al. 2001), we chose to simulate the movements of the fly only phenomenologically. The detailed modelling of the physical properties of the flying blowfly is beyond the scope of this article. For rotations, the SMI generates the yaw velocity as an output signal. For the optomotor controller, the yaw torque is linearly coupled to the yaw velocity. Such a coupling is justified by the very long time constant in the controller, leading to very slow changes in the torque that can be followed by the system even if it is dominated by 
inertia. The same coupling was used in experiments coupling the torque of a tethered fly to the stimulus motion showing that the blowfly can deal with such conditions (Warzecha and Egelhaaf 1996). For the saccadic controller, the time course of the saccade velocity profile of free-flying blowflies (Schilstra and van Hateren 1999) was used as a template to generate realistic turning dynamics. The saccadic controller did not generate any rotations between saccades.

Blowflies show considerable sideward drift after saccades (Schilstra and van Hateren 1999). The observed drift has its likely origin in inertial forces, but may also be controlled actively for steering (Kern and Boeddeker, personal communication). Partial drift compensation presumably results from the fact that blowflies do not fly their saccades by turning around the yaw body axis, but by a combination of yaw, roll, and pitch, leading to a banking behaviour like airplanes show in a sharp turn (Schilstra and van Hateren 1999; van Hateren and Schilstra 1999). We account for the overall drift, irrespective of its origin, by a phenomenological model. Moreover, the fast turns slightly slow down the cyberfly in the heading direction similar as observed in real flies (Kern et al., in preparation), which also needs to be compensated by a thrust control mechanism. To keep our model as simple as possible, we do not propose a thrust control mechanism here. Rather, we lump the different determinants of velocity control into a simple proportional controller. As a further simplification, this controller is assumed to have perfect knowledge of the fly's velocity.

The controller generates a 2D acceleration vector in each time step:

$a_{f}(t)=\frac{1}{\tau_{f}}\left(v_{f}^{d}-v_{f}(t-\Delta t)\right)$

$a_{s}(t)=\frac{1}{\tau_{s}}\left(v_{s}^{d}-v_{s}(t-\Delta t)\right)$

where $a_{f}(t)$ and $a_{s}(t)$ are the forward and sideward components of the acceleration $\vec{a}(t), \vec{v}(t)$ the velocity with its sideward and forward components $v_{s}$ and $v_{f}$, respectively; and $\vec{v}^{d}$ the desired velocity ( $1 \mathrm{~m} / \mathrm{s}$ forward velocity). Sideward drift of different duration and, accordingly, the time it takes for the forward velocity to reach its pre-saccade value are modelled by varying the time constants $\tau_{f}, \tau_{s}$.

For a given time $t$, the velocity is updated by stepwise integration, according to

$\vec{v}(t)=\vec{v}(t-\Delta t)+\vec{a}(t) \cdot \Delta t$

where $\Delta t=1 \mathrm{~ms}$ the duration of a simulation step.

To model the extreme case of perfect velocity control, the velocity was alternatively set to the desired value in each time step

$\vec{v}(t)=\vec{v}^{d}$
The position $\vec{p}(t)$ of the cyberfly is updated according to

$\vec{p}(t)=\vec{p}(t-\Delta t)+\vec{v}(t) \cdot \Delta t$

\section{Results}

3.1 Simulation experiment with different types of SMIs

The performance of a cyberfly was tested with two fundamentally different types of controllers. The first controller (optomotor controller) couples the signals of a major output cell of the blowfly's visual motion pathway, the HSE-cell, in a continuous way to the turning response of the cyberfly. This type of controller implements the classical hypothesis drawn from behavioural optomotor experiments on tethered flying or walking flies with the animal turning on the spot in response to a purely rotating environment [see, e.g., Götz 1975 (Drosophila); Warzecha and Egelhaaf 1996 (Lucilia)]. The second controller (saccadic controller) implements the saccadic flight mode observed in freely flying flies in laboratory (Schilstra and van Hateren 1999; Kern et al., in preparation) and outdoor environments (Boeddeker et al. 2005). This type of controller generates short purely translational flight segments interleaved with sharp fast turns mimicking the body saccades observed in flies. Both controllers and their performance are described in detail together with the performance of the corresponding cyberfly in the following sections.

\subsection{Optomotor controller}

One of the basic behavioural responses observed in many insects is the optomotor turning response. This behaviour is thought to stabilise a straight path of movement by compensating rotations caused either by external disturbances or by internal asymmetries in the motor system. For the horizontal plane, this behaviour can be achieved by balancing out the image velocity the animal observes on both sides (Götz 1975). However, this type of controller has also been proposed to be able, as a useful by-product of the bellshaped ambiguous velocity tuning of the motion detection system (Fig. 3b), to accomplish collision avoidance (Götz 1975). An imbalance of the image velocities can be caused either by self-rotation or by asymmetries in the distance of the environment. Depending on the source of the imbalance, a rotational response to restore optic flow symmetry has to be directed inversely. To compensate for selfrotation, it has to be directed towards the side facing the larger image velocity. When the larger velocity is caused by closer objects, the animal has to generate an avoidance response instead. This reversal in response direction can result from an implicit sign reversal of the differential response caused by 

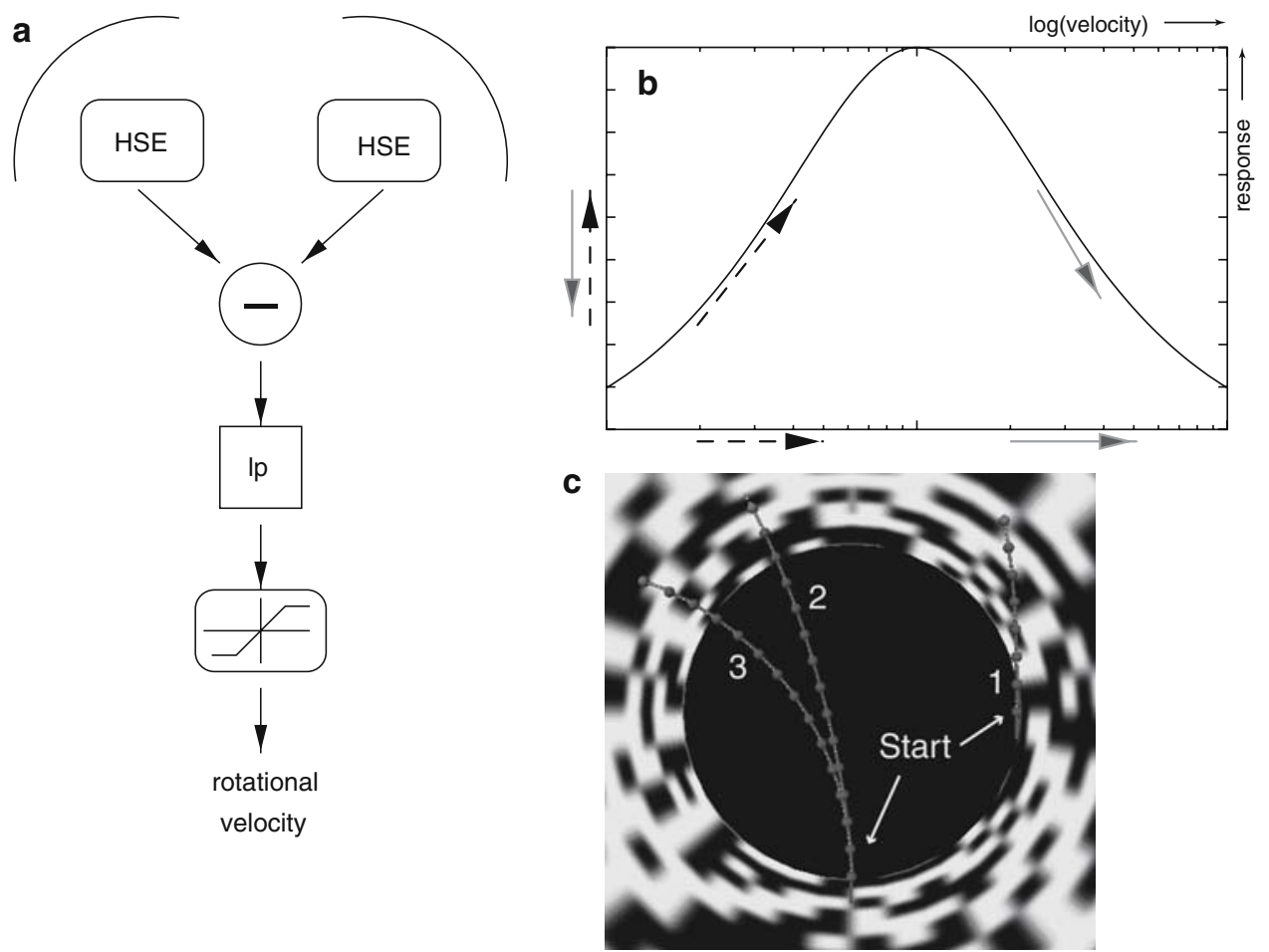

Fig. 3 a The structure of the sensory-motor module implementing the optomotor response. The differential signal of the two HSE input elements of the cyberfly are coupled via a low-pass filter (time constant $750 \mathrm{~ms}$ ) to the rotational velocity the fly generates. The rotational velocity is limited to $3,000^{\circ} / \mathrm{s}$. b The motion detectors used in the sensory model encode the velocity with in bell-shaped dependency. An increase in velocity can thus lead to an increase in response (dashed

the high-velocity translational optic flow when the fly comes close to the arena wall. If the velocities are low, the HSEcell confronted with the faster movement will respond with a larger signal than the cell facing the slower image movement. The situation is inverted if both velocities are beyond the velocity optimum of the movement detectors. In this case, the response of the cell encoding the faster movement will be lower than the response of the cell stimulated with slower motion (Götz 1975). If optomotor behaviour is coupled to the difference of two HSE-cell signals, the sign of the response thus can change depending on the velocity level. A response towards faster motion can turn into an avoidance response simply by increasing the stimulus velocity of an asymmetric translational flow field. A simple optomotor controller couples the difference of two simulated HSE-cells to the yaw torque generated by the system. The simplest way to couple this signal to the yaw torque is a linear coupling defined by a motor gain factor (Fig. 3a).

\subsubsection{Performance of the optomotor controller}

The optomotor controller was tested in two types of simulations. The first and classical experiment is to observe arrows), if the velocities are suboptimal, or to a decrease in response (solid arrows), if the velocities are super optimal. (c) Example trajectories of the closed-loop simulation of this controller: The controller induces a weak tendency to avoid close walls (1) and can counteract a rotational offset ( 2 with, 3 without optomotor response). A top view of the 3D-setup used for all simulation experiments is shown in perspective projection

the optomotor following responses in a system reduced to one rotational degree of freedom. In a second experiment, the behaviour of the system with constant forward velocity was analysed. The simulations were performed in a virtual cylinder with a diameter of $930 \mathrm{~mm}$ and a height of $900 \mathrm{~mm}$ approximated by 36 rectangular wall segments covered with a random dot texture formed by black and white squares with an edge length of $10^{\circ}$ visual angle. The axis of the cylinder is parallel to the yaw axis of the cyberfly; the vertical position of the fly is at half height of the cylinder. If not stated otherwise, the same cylinder is used for all simulations described below.

The optomotor controller was calibrated in the first test situation of the classical optomotor situation with the cyberfly in the centre of a rotating drum. In such a situation, the system is able to control its rotational speed while being fixed in position. The coupling gain was varied systematically. As expected, this SMI showed that the system is not able to compensate for the rotation of the stimulus if the gain is too small. At a critical value of the gain, the system is able to compensate the rotation almost perfectly, leading to a remaining slip velocity close to zero. A gain in this range was used for the subsequent simulations. If the gain is much 
larger than this critical value, the system overcompensates resulting in velocity oscillations around a zero mean.

For the second simulation experiment that was to test whether an optomotor controller can prevent the cyberfly from colliding with the arena wall, the cyberfly was moved with a constant velocity of $1 \mathrm{~m} / \mathrm{s}$, which is in the range of translation velocities of blowflies in arenas of comparable size (Kern, in preparation). The gain was chosen as determined as optimal in the classical optomotor situation (first experiment). A cyberfly with an optomotor controller inherently shows, as proposed by Götz (1975), a tendency to avoid an approaching wall in a cylindrical test environment (Fig. 3c, trajectory 1). However, this turning tendency was by far too weak to prevent the cyberfly from colliding with the wall. This finding is qualitatively invariant for a large range of gain factors. By systematic variation we did not find a gain factor leading to a sufficient wall avoidance of a stable system.

The optomotor response is supposed to serve the stabilisation of a straight translatory movement and to correct for external rotatory disturbances and asymmetries in the flight motor or walking apparatus. Consistent with other technical implementations (Harrison and Koch 1999; Webb et al. 2004), the system is able to correct for a motor bias in a combination of translatory and rotatory self-motion. In the example shown in Fig. 3c the cyberfly was translated with a constant forward velocity of $1 \mathrm{~m} / \mathrm{s}$. A superimposed rotatory offset of $100^{\circ} / \mathrm{s}$ simulated the motor bias. The system compensates for the rotatory offset to a large extent, which leads to a larger radius in the resulting trajectory (Fig. 3c, trajectory 2) than would result from the uncorrected bias (Fig. 3c, trajectory 3 ). However, the system did not show any pronounced collision avoiding tendency.

If the sign of coupling is inverted, the optomotor response drives the system on a circular trajectory, because the turning response to a small asymmetry in the input increases the asymmetry in this situation. Although the non-linear velocity dependence of the motion detection system prevents an infinite angular acceleration, the system is trapped in its circular movement.

\subsection{Saccadic controller}

Although flies show optomotor responses that may serve course stabilisation against external disturbances or internal asymmetries of the flight apparatus, it is obvious that other visually guided control systems are required to account for collision avoidance. The cyberfly shows, at least under some environmental conditions, wall avoidance behaviour when introducing a saccadic motion pattern reminiscent of freely flying blowflies. This strategy effectively separates the translational from the rotational components of the optic flow. In this part of the analysis, the properties of the saccadic system were evaluated.

\subsubsection{Organisation of the saccadic controller}

The saccadic controller basically forms a state machine with 3 distinct states (Fig. 4a). The generation of a saccadic turn forms one state (called S1 in the following). The duration of $\mathrm{S} 1(23-70 \mathrm{~ms})$ depends on the amplitude of the generated saccade. After generating the turn, the controller switches to state $\mathrm{S} 2$ of saccadic suppression during which no turn is generated. After a fixed duration $(20 \mathrm{~ms})$ of $\mathrm{S} 2$, the controller switches to state I in which no rotational movement is generated and the cyberfly generates only translatory movements. During the purely translatory flight in state I, thresholding is applied to the HSE signals on each side of the cyberfly (Fig. 4c). When one of the HSE signals exceeds the threshold, the controller switches to state S1 again, initiating the next saccadic turn. The threshold value declines exponentially over time during state I (time constant of decline $40 \mathrm{~ms}$ ). Starting from a depolarisation value above the dynamic range of the HSE signals it asymptotically reaches a value below the typical depolarisation of HSE observed during straight flight in the test arena. This exponential decay modulates the probability of generating the next saccade: The longer the intersaccadic interval lasts, the more likely the generation of the next saccade becomes. For a given distance of the cyberfly to objects or walls, this also leads to a regular generation of saccades within similar intervals, as observed in free blowfly flight (Kern et al., in preparation), without explicitly modelling an internal clock for saccade generation. With the transition from state $\mathrm{S} 2$ to state $\mathrm{I}$, the threshold value is reset.

In state $\mathrm{S} 1$, the controller generates the yaw velocity of the saccade according to the experimentally determined template (Fig. 4b, see Schilstra and van Hateren 1999). The saccade is directed towards the side of the HSE-cell that is more depolarised, i.e., the one where the threshold is exceeded first. Caused by the pattern dependencies and the non-linear velocity tuning of the correlation motion detectors this can mean that the other HSE neuron experiences the larger optic flow (see Fig. 3b, solid arrows). Our results indicate that this is indeed the case in the tested environment, since the cyberfly is successful in avoiding the wall and thus turns away from the side experiencing the larger optic flow. For the generation of saccades, a fixed motor program is assumed to generate a stereotypical velocity time course of the cyberfly in every saccade. The fly modulates both the duration and the amplitude of the velocity time course to achieve different turning angles (Fig. 4a). The saccade amplitude is determined by the normalised difference response of the right (R) and left (L) HSE-cell at the time when the saccade is evoked:

$d_{L R}=C \frac{|L-R|}{|L|+|R|}$ 
Fig. 4 a The saccadic controller is based on a simple state machine. Rounded boxes represent states and the arrows indicate the conditioned state changes. b The time course of saccadic turns generated in State $\mathrm{S} 1$. Yaw rotational velocity of saccades from recorded free flight trajectories (Schilstra and van Hateren 1999) were averaged over classes of saccade amplitudes. Classes are given in the plot legend defined by the peak velocity in a saccade. c Structure of the saccadic controller. An adaptive threshold (decay time constant $40 \mathrm{~ms}$ ) is applied to the HSE signals. HSE signals above threshold trigger a saccade. The amplitude is determined from the contrast of the HSE signals at trigger time, the direction determined by which side triggered the saccade. The pattern generator selects the appropriate template from (b) based on the calculated amplitude
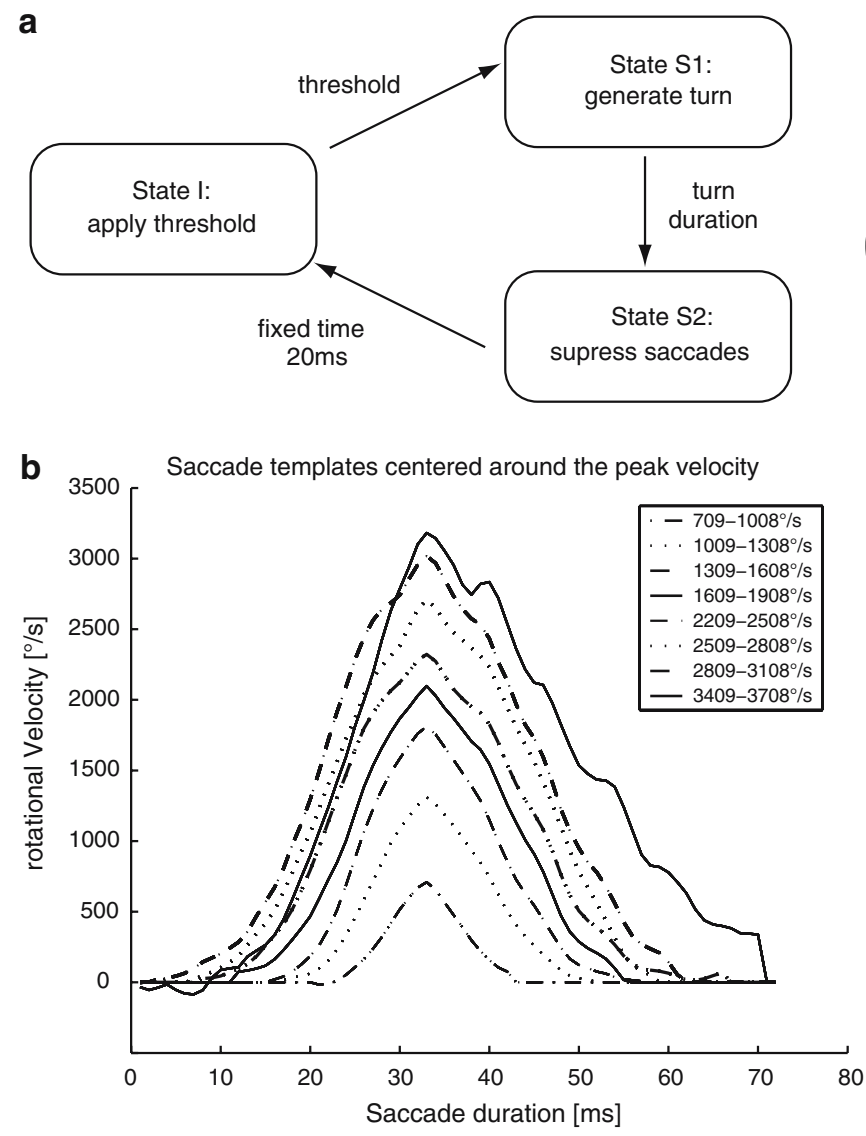

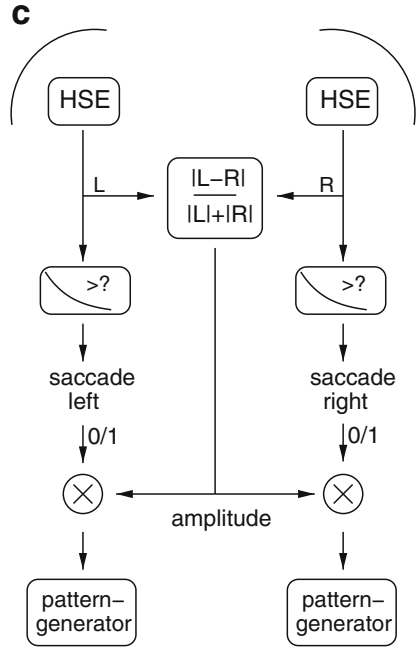

with $C=10$ being a scaling factor determined by systematic variation to map the relevant range of HSE contrast values to the number of saccade templates.

The amplitude $a_{s}$ of the saccade is interpreted as the index to the $N=8$ velocity templates (Fig. $4 \mathrm{~b}$ ) derived from blowfly free flight data (Schilstra and van Hateren 1999):

$a_{s}=\left\{\begin{array}{ll}N-1 & \text { if } d_{L R} \geqslant N \\ \left\lfloor d_{L R}\right\rfloor & \text { otherwise }\end{array}\right.$.

Although insects including blowflies modulate their flight velocity depending on the environmental structure (Srinivasan and Zhang 2000; Kern et al., in preparation), we simplified the controller in this respect. The desired value for proportional control of the velocity is a constant forward velocity of $1 \mathrm{~m} / \mathrm{s}$.

The properties of the saccadic controller were evaluated for different time constants of sideward drift and forward velocity control; i.e., several values for $\tau_{f}, \tau_{s}$ were tested (Eq. 2; Fig. 5). The starting conditions and the turn templates (e.g., Fig. 5a) were identical. In the special case without drift (Eq. 4) the trajectory is always tangential to the cyberfly orientation (Fig. 5b). For large time constants (i.e., $\tau_{s}=$ $\tau_{f}=50 \mathrm{~ms}$ ), pronounced drift movements are generated resulting in an initial turn with very large radius (Fig. 5d).
Intermediate drift compensation (e.g., $\tau_{s}=\tau_{f}=10 \mathrm{~ms}$ ) leads to sideward drift as is typically observed in blowfly flight (Fig. 5e). To test the effects of the time constant of sideward drift decay and deceleration following a turn we also parameterised the two controllers with different time constants. Figure $5 \mathrm{c}$, f shows examples for the resulting turn dynamics. Note, that the cyberfly accelerates during the turn if the forward deceleration is compensated more quickly than the sideward drift decays (i.e., $\tau_{s}>\tau_{f}$, Fig. 5f). This is in contradiction to observations in free blowfly flight (Kern et al., in preparation), making these parameter constellations biologically implausible.

Figure 6 further illustrates the resulting sideward drift movements present in the trajectories resulting from the velocity controller.

\subsubsection{Saccadic controller without sideward drift}

In a first version, the saccadic controller compensates completely for any sideward drift (Eq. 4). In the resulting trajectory the cyberfly is always oriented tangentially to its flight path (Fig. 6a). Without sideward drift, the saccadic flight mode leads to a much better avoidance of the cylinder walls in the test environment than the optomotor controller. Hence, 
Fig. 5 Turn trajectories generated by different thrust control time constants for a given turn template starting from identical conditions. a The rotational velocity generated by the saccadic controller. b For complete compensation of drift and deceleration after the turn.

The cyberfly orientation is always parallel to the direction of flight. c-f If the velocity effects of the turn are compensated by the proportional controller, the cyberfly drifts into the previous direction of flight after the turn. Different combinations of values result in different turn dynamics (c: $\tau_{f}=50 \mathrm{~ms}, \tau_{s}=10 \mathrm{~ms}$, d : $\tau_{f}=50 \mathrm{~ms}, \tau_{s}=50 \mathrm{~ms}$, e : $\tau_{f}=10 \mathrm{~ms}, \tau_{s}=10 \mathrm{~ms}$, f : $\tau_{f}=10 \mathrm{~ms}, \tau_{s}=50 \mathrm{~ms}$ ) a
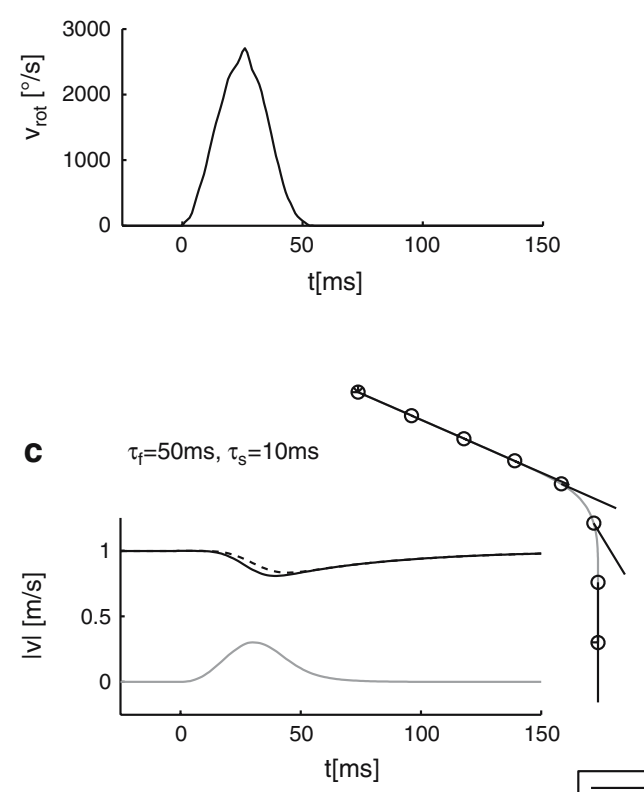

\begin{tabular}{|r|}
\hline fwd \\
swd \\
---- abs \\
\hline
\end{tabular}

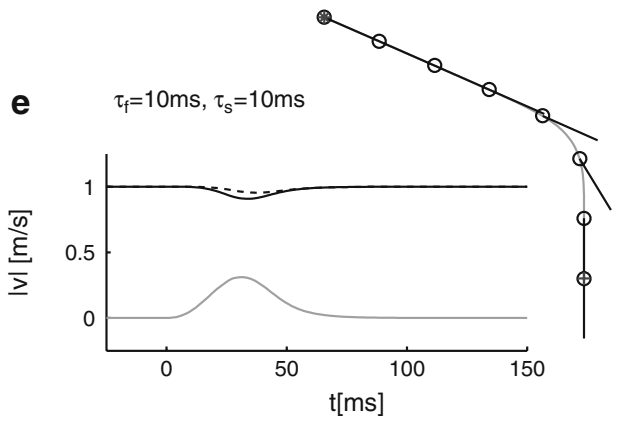

d $\quad \tau_{\mathrm{f}}=50 \mathrm{~ms}, \tau_{\mathrm{s}}=50 \mathrm{~ms}$

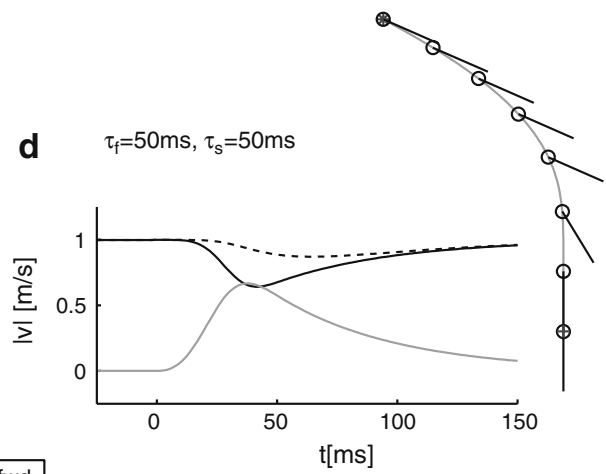

b veconst.
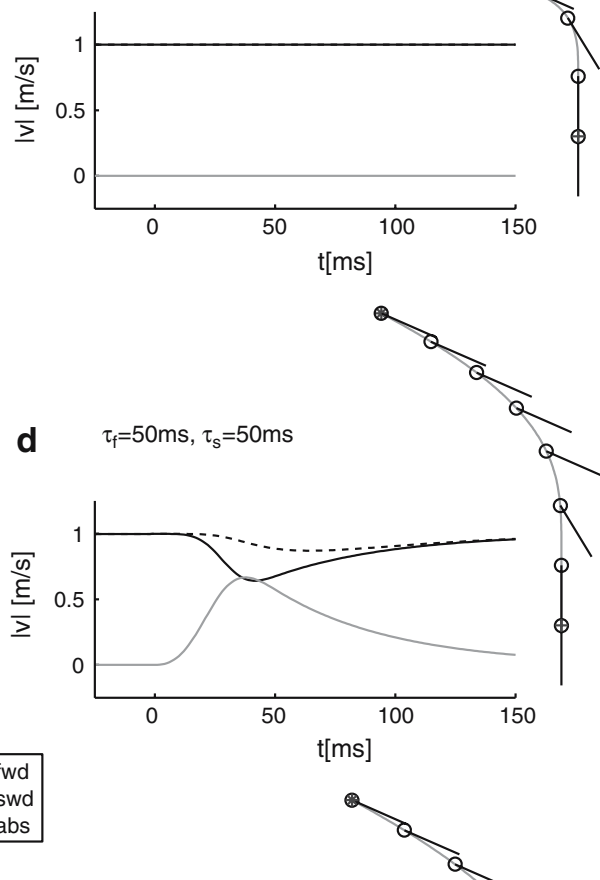

f $\quad \tau_{\mathrm{f}}=10 \mathrm{~ms}, \tau_{\mathrm{s}}=50 \mathrm{~ms}$

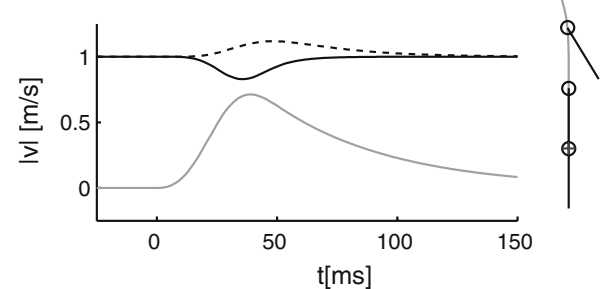

a saccadic flight strategy, as observed in flies, appears to facilitate wall avoidance. Nonetheless, wall avoidance is not fully reliable and depends on the starting position of the cyberfly. This is illustrated by the length of flight trajectories starting at different locations in the flight arena (Fig. 7). The cyberfly always started in the direction indicated by the arrow in Fig. 6. The maximal flight duration was limited to a $9.75 \mathrm{~s}$ test period (initial $5 \mathrm{~s}$ shown in Fig. 6). The maximal path length that can be reached at a translation velocity of $1 \mathrm{~m} / \mathrm{s}$ within this time without collision with the arena wall amounts to $9.75 \mathrm{~m}$. For some starting positions, the cyberfly crashes into the arena wall after a shorter period of time.

\subsubsection{Systematic variation of sideward drift}

Although a saccadic controller with no sideward drift is quite successful in avoiding collisions with the arena wall, blowflies show a pronounced sideward drift after saccades
(Schilstra and van Hateren 1999). To assess the consequences of different amounts of sideward drift, we varied the time constants for forward and sideward velocity control in the range of $10 \mathrm{~ms} \leqslant \tau \leqslant 50 \mathrm{~ms}$. For larger values than $50 \mathrm{~ms}$, the velocity effects of one saccade last beyond the following intersaccadic interval, leading to instabilities in the system. System performance was determined by the distance travelled on average for 81 different starting positions in the cylinder for each parameter constellation (see Fig. 7 for exemplary flight length histograms). Figure 8 shows how the average flight length before colliding with the arena wall depends on the two time constants, which were varied in steps of $10 \mathrm{~ms}$ in the range between 10 and $50 \mathrm{~ms}$. Shorter time constants would be implausible for a neuronal control mechanism, and for larger values the system would not regain the set velocity within the intersaccadic interval. Figure 6b, c shows examples of the trajectories for the extreme parameter settings. The maximal flight distance of $7.8 \mathrm{~m}$ is reached 

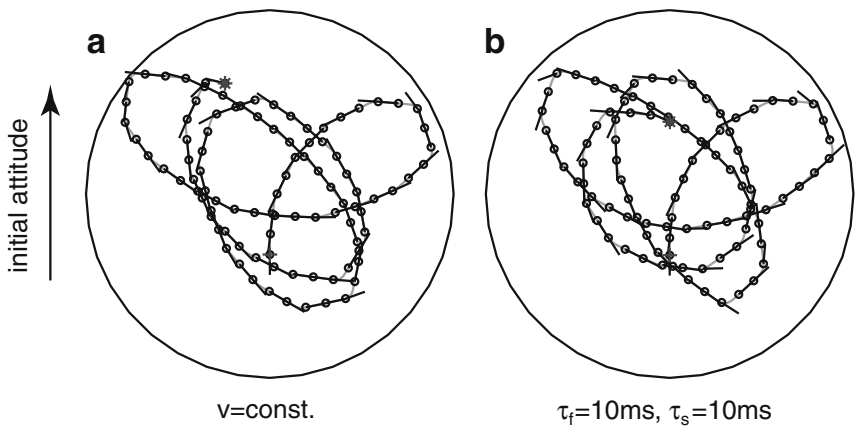

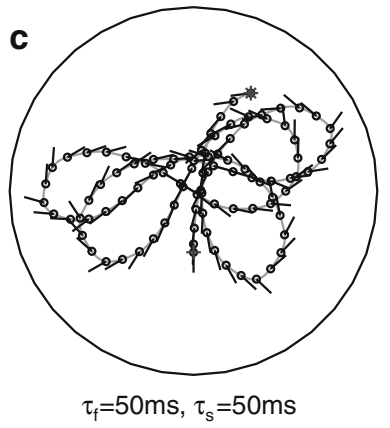

flight. b, c Two parameter constellations for the proportional velocity controller shown exemplarily $\left(\mathbf{b}: \tau_{f}=10 \mathrm{~ms}, \tau_{s}=10 \mathrm{~ms}, \mathbf{c}: \tau_{f}=\right.$ $50 \mathrm{~ms}, \tau_{s}=50 \mathrm{~ms}$ ). Note that the trajectories for larger time constants appear smoother, although the rotational dynamics are unchanged

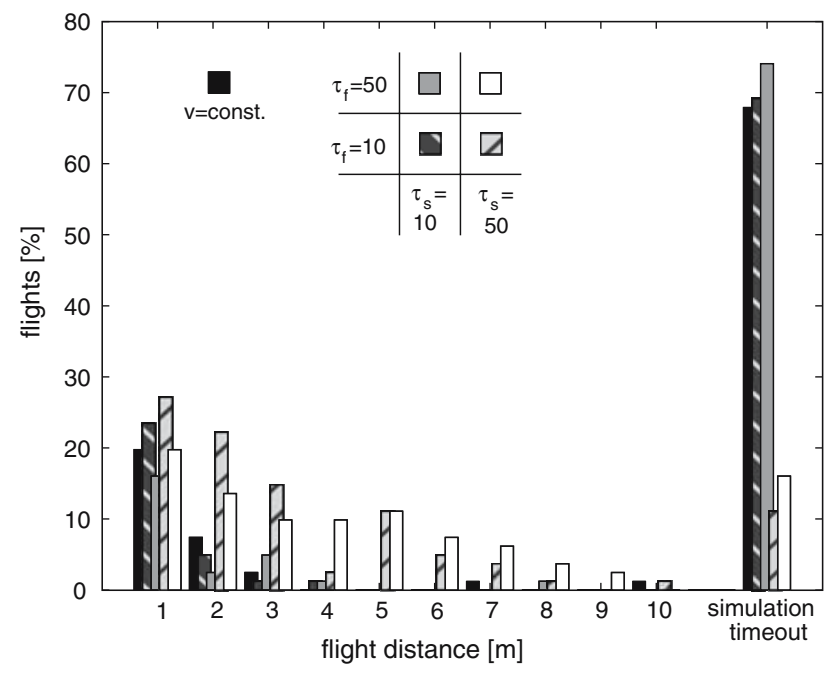

Fig. 7 Histogram showing the distribution of flight lengths for five parameter constellations (see legend). Bars show percentages of flights from 81 different starting positions. Flight lengths for each condition were binned into 10 bins. Most flights are ended by a simulation timeout for low sideward time constants $\left(\tau_{f}<20 \mathrm{~ms}\right.$ or $v=$ const). For large values $\left(\tau_{s}=50 \mathrm{~ms}\right.$ ), flight lengths are almost equally distributed

for a parameter constellation at the boundary of this range $\left(\tau_{s}=10 \mathrm{~ms}, \tau_{f}=50 \mathrm{~ms}\right)$, the flight distance of a cyberfly without any sideward drift is $7.2 \mathrm{~m}$ on average. In comparison, this suggests that the flight performance slightly increases for small time constants of sideward drift decay. However, larger time constants of sideward drift decay have a clear negative effect on the performance. The time constant of the forward velocity controller has only a weak effect on the performance for a given value for the sideward controller.

The near columnar structure of the contour plot (Fig. 8) shows that the effect of the time constant of the sideward drift decay is strong. The effect of a larger time constant for forward velocity control following a turn is much less prominent.

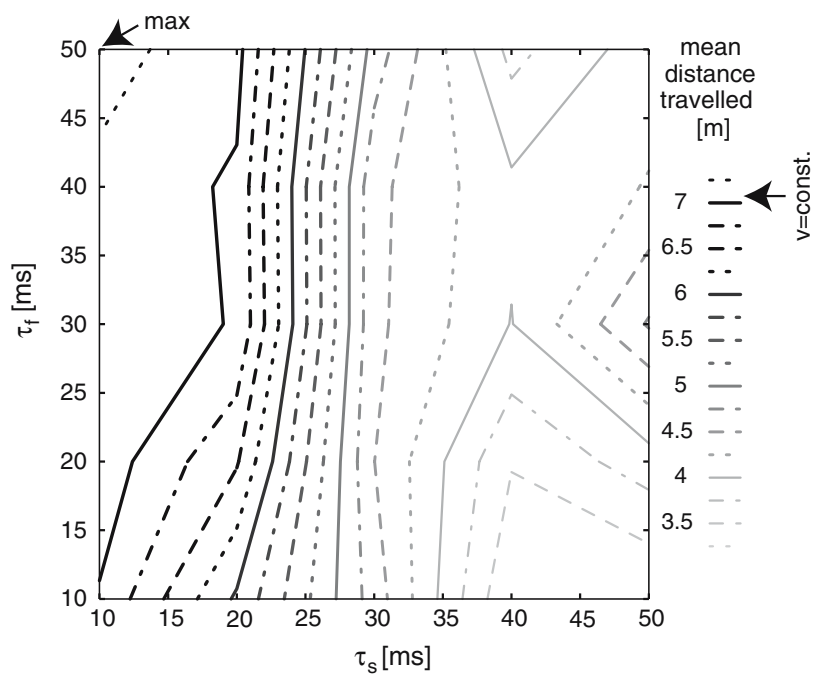

Fig. 8 Dependency of cumulated flight distance on the controller parameters. The arrow indicates the performance of the controller generating constant forward velocity

\subsubsection{Simple additional rule for straight wall approaches}

We observed that the cyberfly with saccadic controller as discussed above often fails when the wall is approached headon. This is plausible since such an approach leads to a virtually symmetrical optic flow across the two eyes, inducing only a negligible difference in the HSE responses. Accordingly the generated saccade amplitude (Eqs. 6, 7) is small even for large velocities in the resulting expansion flow field. As a result the cyberfly generates small saccades at a high frequency when approaching the wall perpendicularly on a straight line, leading to an insufficient avoidance response. For a starting position close to the wall and a radial initial direction the cyberfly fails to generate a sufficiently large saccade (Fig. 9a). 


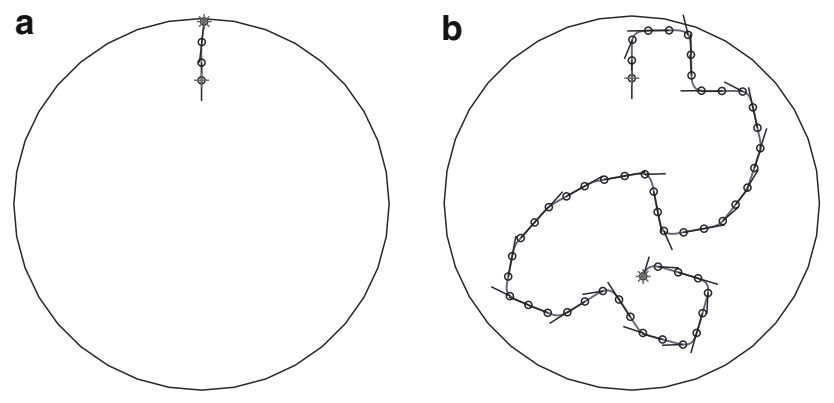

Fig. 9 Two trajectories with the same starting condition illustrating the effect of a simple change in saccade generation rules: a Small HSE contrast values lead to small saccade amplitudes (Eq. 6), the system fails in a straight approach to the wall. b When large saccades are generated for symmetrical HSE activity, the wall avoidance is successful

To overcome this problem we extended the saccade generation mechanism by a simple additional rule to determine the saccade amplitude

$a_{s}^{\prime}=\left\{\begin{array}{cc}N-1 & \text { if } a_{\mathrm{s}}=0 \\ a_{s} & \text { otherwise }\end{array}\right.$

The system, thus, generates a large saccadic amplitude for almost symmetrical excitation of the simulated neurons $\left(d_{L R}<1 \Rightarrow a_{s}=0\right) . a_{s}^{\prime}$ then replaces the smallest saccadic template with the largest one. Note, this rule is only based on the ad hoc consideration that a large saccade should be generated when the fly approaches a wall approximately perpendicularly and is so close that image expansion velocity is large.

The result of this additional rule is illustrated by Fig. $9 \mathrm{~b}$. The system generates a large initial saccade avoiding the wall. Note that the cyberfly now generates large saccades also close to the centre of the arena, when a small difference in the HSE signals is encountered, a finding of the model simulation that is not in contradiction to experimental results. In any case, the simple extension of the saccadic controller leads to a much better performance as illustrated by the flight length histogram shown in Fig. 10. With this extension the cyberfly collides with the arena wall in only very few cases.

\subsubsection{Dependence on the wall texture}

It is well known that the responses of the correlation type motion detector and of the postsynaptic neurons integrating their responses strongly depend on the textural properties of a moving pattern (Buchner 1984; Dror et al. 2001; Eckert and Hamdorf 1981). To test whether the wall avoidance behaviour of the saccadic controller is robust against changes in texture, we tested the system for different wall textures. Figure 11 shows exemplarily the result of a simulation experiment $\left(\tau_{s}=\tau_{f}=10 \mathrm{~ms}\right)$ with the texture $\left(\mathrm{T}_{1}\right)$ used for the calibration of the cyberfly in comparison to a tex-

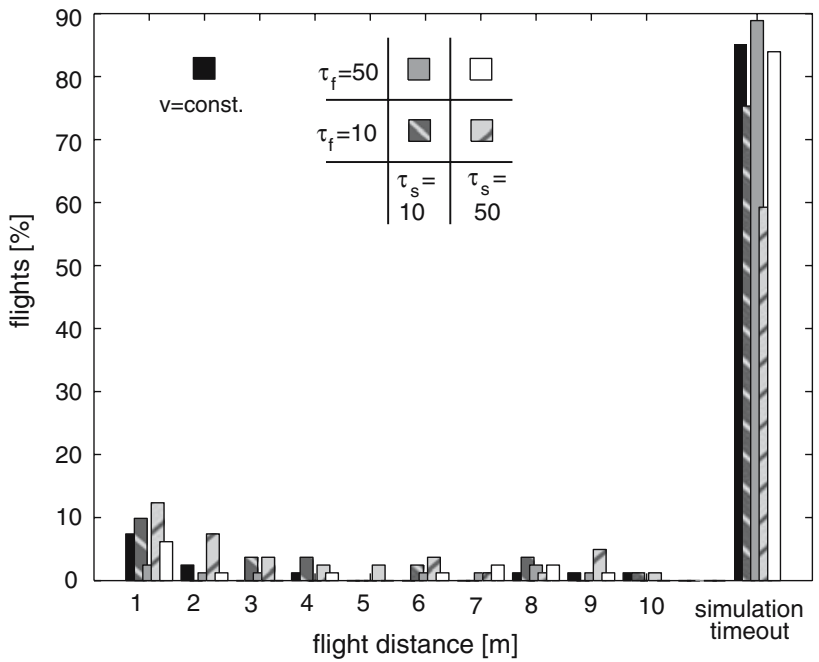

Fig. 10 Histogram of flight lengths for the saccadic controller with an additional rule to generate large saccades under symmetrical conditions. Bars show percentages of flights from 81 different starting positions. Compare to Fig. 7

ture $\left(\mathrm{T}_{2}\right)$ generated downscaling of the pattern. Although this change in pattern cause only a minor change in the position of the velocity optimum in the steady-state tuning of the simulated HSE cell, the controller generates differently directed initial saccades from identical starting conditions. While the simulated flight in the arena textured with $T_{1}$ is ended by a timeout criterion for most starting positions (Fig. 10), the cyberfly crashes into the arena wall for all tested starting conditions with texture $T_{2}$. The wall avoidance behaviour achieved for texture $T_{1}$ cannot be regained for texture $T_{2}$ by adjusting the controller parameters.

\section{Discussion}

This paper employs a modelling approach to investigate the potential functional significance of the strategy of saccadic flight and gaze control which is a distinguishing feature of spontaneous flight behaviour of blowflies (Schilstra and van Hateren 1999; van Hateren and Schilstra 1999). In previous studies we could show that a population of output neurons in the blowfly visual motion pathway extracts information about all self-motion components from the complex optic flow patterns generated on the eyes while the blowfly is flying around in its environment (Boeddeker et al. 2005; Karmeier et al. 2006; Kern et al. 2005; van Hateren et al. 2005). In the latter accounts the blowfly's brain is concluded to use a saccadic gaze strategy to obtain information between saccades about the spatial layout of the environment. The low-frequency membrane potential modulations between saccades provide information about the translational optic flow components 
Fig. 11 Texture dependent change in performance.

Exemplary trajectories (saccadic controller with

symmetry-braking extension, $\tau_{f}=10 \mathrm{~ms}, \tau_{s}=10 \mathrm{~ms}$ ) for two random checkerboard patterns constructed from differently sized texels. Starting conditions are identical despite from the texture. a Texture $\mathrm{T}_{1}$ used for statistics shown in Fig. 10. b Texture $T_{2}$ generated by repetition and scaling of the same pattern
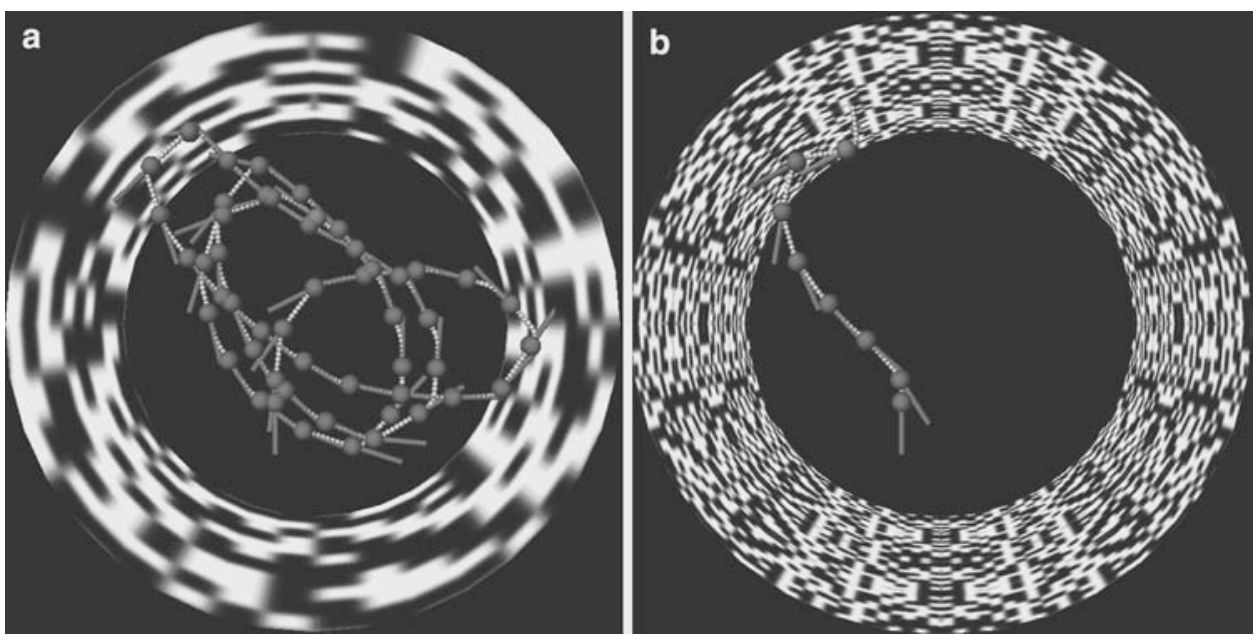

which are, compared to the rotational flow component, much larger during these flight segments than during the saccades (Kern et al. 2005). The translational optic flow is particularly relevant from a functional point of view, because it provides relative distance information about the three-dimensional structure of the environment (Koenderink and van Doorn 1987) and, most likely, represents the only source of spatial information available for an airborne blowfly.

Although the above-mentioned studies show that spatial information is represented by the visual system between saccades, they do neither tell us how this information can be extracted nor whether this information is really sufficient to control the blowfly's flight behaviour and, in particular, to prevent the blowfly from colliding with obstacles. This problem was approached here by developing a cyberfly that is provided with visual information by mechanisms that mimic those of the real blowfly. The cyberfly uses this information to successfully avoid obstacles in a virtual environment in most cases.

The cyberfly, as presented here, solves only one out of the many visually guided behavioural tasks of a real fly. This first version is extremely simplified compared with its biological counterpart: (1) It controls movements only in a plane, whereas real flying blowflies can, of course, move in all three dimensions and thus have to control six degrees of freedom. (2) We only took into account one type of output neuron of the blowfly visual system, the HSE-cell. Being an output neuron of the system and sensitive to horizontal movement (Hausen 1984), HSE is probably one of the most important neurons involved in controlling horizontal turns. Nonetheless, several additional neurons are likely to be involved in visual flight control, especially if all degrees of freedom of locomotion are taken into account (Karmeier et al. 2006). (3) The forward velocity generated by the cyberfly was held constant, although recent observations suggest that blowflies modify their forward velocity depending on the environmental prop- erties (Kern et al., in preparation). A similar behaviour was observed previously in bees (Srinivasan et al. 1996). (4) The physical constraints of the blowfly flight were not taken into account for this study. Instead, a phenomenological approach was taken by controlling the velocity with a proportional control scheme.

While the proposed simple version of a cyberfly successfully avoids collisions with the wall of the flight arena in most situations, it fails reproducibly when approaching a wall straight ahead. This is plausible since such an approach should lead to an almost symmetrical activity in the two sensory input neurons which is transformed to only small saccade amplitudes by the saccadic controller. The generation of large saccades in response to strong frontal expansion flow, as discussed recently for Drosophila (Bender and Dickinson 2006) could solve this problem. Similar mechanisms to avoid frontal expansion were also proposed (Neumann 2004) and successfully tested on robots (Webb et al. 2004). The generation of large saccades when approaching a wall perpendicularly can also be accomplished in our cyberfly with a simple extension of the transformation of the HSE difference signal into saccade amplitude. If large saccades are generated when the difference signal of the two HSE-cells is small, the cyberfly can also successfully prevent obstacle collision during a perpendicular approach of a wall.

Our analyses revealed another severe limitation of our current simple version of a cyberfly, i.e., the dependence of its performance of the textural properties of the arena. The texture dependence of local movement detectors of the correlation type and of the motion induced response of fly tangential cells is well known (Buchner 1984; Dror et al. 2001; Eckert and Hamdorf 1981). Nevertheless it seems very unlikely that a fly would fail to avoid the walls of a cylindrical setup with any of the tested textures, although there is evidence that they change their flight pattern and velocity when the environmental properties are altered (Frye and Dickinson 2007; 
Kern et al., in preparation). The control of the forward flight speed based on the responses of optic flow processing neurons (see below) may be the key to a more robust behaviour. We will analyse this in more detail in a forthcoming study.

Despite the simplicity of our approach, the closed-loop model simulations clearly show that a saccadic flight strategy helps, at least under specific environmental conditions, the cyberfly to avoid collisions with the wall of our flight arena. This behaviour of a suitably parameterised saccadic controller is in stark contrast to the performance of an optomotor controller. We could show that, in contrast to an older hypothesis (Götz 1975), the smooth and continuous optomotor controller is incapable of controlling wall avoidance in our setup, even if it is parameterised to create a realistic optomotor following response to pure rotational stimuli. However, in accordance with previous studies (Webb et al. 2004), such a controller can compensate for systematic asymmetries of the motor system. For the path stabilisation task, the optomotor circuit may very well be active in the fly evaluating the optic flow in parallel to a visual saccadic controller.

All sensory-motor interfaces of the cyberfly tested in the present study had in common that, in contrast to previous modelling attempts (see Sect. 1), we used a model of the blowfly visual motion pathway as sensory input stage that was parameterised to account for the complex timedependent responses of the HSE-cell of real blowflies to behaviourally generated optic flow (Lindemann et al. 2005), and that we took the dynamical characteristics of free flight of blowflies into account. In particular, we analysed the significance of sideward drift after saccadic turns that is one of the most distinguishing features of blowfly flight behaviour. The sideward components of head and body movements were concluded to be encoded in the responses of HSE neuron (Karmeier et al. 2006; Kern et al. 2005, 2006). Sideward movements could be useful in the context of obstacle avoidance, if the blowfly is oriented perpendicular to an obstacle. In this case pure forward translation would not lead to an asymmetric response in the HSE neurons in both halves of the visual system, whereas sideward movements could lead to asymmetric responses.

The performance of the cyberfly with a saccadic controller was found to depend strongly on the strength of sideward drift after saccades. Although Calliphora HSE signals were concluded to provide information about sideward drift, the cyberfly is relatively successful in avoiding collisions with the arena wall without sideward drift after saccades. This finding is in accordance with a previous study to model Drosophila flight behaviour (Reiser and Dickinson 2003). This similarity in performance is interesting, because the Drosophila model differs from our cyberfly in at least two relevant ways. (1) The sensory module of the Drosophila model is based on some sort of hypothetical expansion detector, whereas at least the sensory module of the basic version of our saccadic cyberfly is modelled after an identified output neuron of the blowfly which strongly responds to optic flow resulting from translatory movements between saccades. (2) The saccade amplitude of the Drosophila model is determined according to a random distribution independent of the strength of the optic flow, while only the timing and direction of saccades depend on the visual motion input. This is quite different for our cyberfly where also the saccade amplitude depends on the difference of the responses of HSE-cells in both halves of the brain. Although a cyberfly without sideward motion may be appropriate to account for flight behaviour of the small Drosophila, it cannot account for the much heavier blowflies, because they show pronounced sideward movements after saccades (Schilstra and van Hateren 1999). We could show that a saccadic controller with sideward drift with a short time constant is slightly superior to a controller without sideward drift. A sideward drift component between saccades may allow the cyberfly to extract the distance information to frontal obstacles from the translational optic flow. Similar mechanisms have been investigated in the context of sideward peering in locusts and preying mantis (Kral et al. 2000; Sobel 1990). In accordance with a previous study (Karmeier et al. 2005), intersaccadic intervals of some ten milliseconds are sufficiently long for providing the controller with behaviourally relevant optic flow information.

The cyberfly has some free parameters in addition to the time constant of drift decay. These are the time constant of the threshold decrease, the initial and asymptotic value of the threshold, the scaling factor of the saccade amplitude, the thrust control time constants $\tau_{f}, \tau_{s}$, and the duration of saccade suppression. Amongst these, the control time constant for the sideward component of the velocity appears to be the most decisive one with respect to wall avoidance performance.

The current version of the cyberfly is completely deterministic. No noise is added to any stage of the control loop. However, small variations in the texture, the starting positions, or the parameters (especially the sideward thrust amplitude) revealed that even tiny differences in the initial input signals can accumulate to large differences in the final trajectory after some seconds of closed-loop simulation, even if the initial HSE signals are virtually the same. The introduction of noise would probably make the trajectories more independent of the textural details of the environment, but would necessitate statistical analysis of a large number of simulation runs multiplying the computational effort for parameter optimisation. Adding noise to the sensory part of the model would be straightforward because the noise statistics for the HSE neurons simulated in that module are well known (Warzecha et al. 1998). Some of the noise effects could be reduced by applying a lowpass filter to the HSE signals before further processing, but this would add at least one further parameter. 
Although our basic version of the cyberfly with sideward drift as observed in real blowflies is already relatively successful in obstacle avoidance, it performs still much worse than the real blowfly. Therefore, the model needs to be elaborated in various ways. One elaboration has been proposed already in the present study by a modified transformation of the difference signal of the HSE-cells into saccade amplitude. This modification is, however, a purely formal one without recourse to experimentally determined neuronal properties. However, more sophisticated representations of optic flow parameters may be obtained in a neuronally plausible way by using the signals of additional optic flow sensors, such as the other HS-cells and the vertically sensitive VS-cells. These cells could be used by the saccade controller to provide information about self-motion in three dimensions and to control other flight parameters such as thrust, pitch or height above the simulated ground.

Acknowledgements This work was supported by the Deutsche Forschungsgemeinschaft (DFG).

\section{References}

Bender JA, Dickinson MH (2006) Visual stimulation of saccades in magnetically tethered Drosophila. J Exp Biol 209:3170-3182

Boeddeker N, Egelhaaf M (2003) Steering a virtual blowfly: simulation of visual pursuit. Proc R Soc Lond B 270:1971-1978

Boeddeker N, Kern R, Egelhaaf M (2003) Chasing a dummy target: smooth pursuit and velocity control in male blowflies. Proc R Soc Lond B 270:393-399

Boeddeker N, Lindemann JP, Egelhaaf M, Zeil J (2005) Responses of blowfly motion-sensitive neurons to reconstructed optic flow along outdoor flight paths. J Comp Physiol A 25:6435-6448

Borst A (2004) Modelling fly motion vision. In: Feng J (ed) Computation neuroscience: a comprehensive approach. Chapman \& Hall/CTC, London pp 397-429

Borst A, Egelhaaf M, Haag J (1995) Mechanisms of dendritic integration underlying gain control in fly motion-sensitive interneurons. J Comput Neurosci 2:5-18

Borst A, Haag J (2002) Neural networks in the cockpit of the fly. J Comp Physiol A 188:419-437

Borst A, Reisenman C, Haag J (2003) Adaptation to response transients in fly motion vision: II. Model Stud Vis Res 43:1309-1322

Buchner E (1984) Behavioural analysis of spatial vision in insects. In: Ali MA (ed) Photoreception and vision in invertebrates. Plenum Press, New York pp 561-621

Chahl JS, Srinivasan M (2000) A complete panoramic vision system, incorporating imaging, ranging, and three dimensional navigation. In: Proceedings of the IEEE Workshop on Omnidirectional Vision, pp 104-111

Dahmen HJ, Wüst RM, Zeil J (1997) Extracting egomotion parameters from optic flow: principal limits for animals and machines. In: Srinivasan MV, Venkatesh S (eds) From living eyes to seeing machines. Oxford University Press, Oxford, pp 174-198

Dror RO, O'Carroll DC, Laughlin SB (2001) Accuracy of velocity estimation by Reichardt correlators. J Opt Soc Am A 18:241-252

Eckert H, Hamdorf K (1981) The contrast frequency-dependence: a criterion for judgung the non-participation of neurones in the control of behavioural responses. J Comp Physiol A 145:241-247
Egelhaaf M (2006) The neural computation of visual motion information. In: Warrant E, Nilsson D-E (eds) Invertebrate vision. Cambridge University Press, Cambridge, pp 399-461

Franceschini N, Pichon JM, Blanes C (1992) From insect vision to robot vision. Phil Trans R Soc Lond B 337:283-294

Franz MO, Chahl JS, Krapp HG (2004) Insect-inspired estimation of egomotion. Neural Comput 16:2245-2260

Franz MO, Mallot HA (2000) Biomimetic robot navigation. Robot Auton Syst 133-153

Frye MA, Dickinson MH (2007) Visual edge orientation shapes freeflight behavior in drosophila. Fly 1:e1-e2

Götz KG (1975) The optomotor equilibrium of the Drosophila navigation system. J Comp Physiol 99:187-210

Harrison RR, Koch C (1999) A robust analog VLSI motion sensor based on the visual system of the fly. Auton Robot 7:211-224

Hausen K (1982a) Motion sensitive interneurons in the optomotor system of the fly. I. The horizontal cells: structure and signals. Biol Cybern 45:143-156

Hausen K (1982b) Motion sensitive interneurons in the optomotor system of the fly. II. The horizontal cells: receptive field organization and response characteristics. Biol Cybern 46:67-79

Hausen K (1984) The lobula-complex of the fly: structure, function and significance in visual behaviour. In: Ali MA (ed) Photoreception and vision in invertebrates. Plenum Press, New York, pp 523-559

Hausen K (1993) Decoding of retinal image flow in insects. In: Miles FA, Wallman J (eds) Visual motion and its role in the stabilization of gaze. Elsevier, Amsterdam, pp 203-235

James AC (1990) White-noise studies in the fly lamina. Ph.D. Thesis, Australian National University, Canberra

Juusola M, Uusitalo RO, Weckström M (1995) Transfer of graded potentials at the photoreceptor-interneuron synapse. J Gen Physiol 103:117-148

Karmeier K, Krapp HG, Egelhaaf M (2005) Population coding of selfmotion: applying Bayesian analysis to a population of visual interneurons in the fly. J Neurophysiol 94:2182-2194

Karmeier K, van Hateren JH, Kern R, Egelhaaf M (2006) Encoding of naturalistic optic flow by a population of blowfly motion sensitive neurons. J Neurophysiol 96:1602-1614

Kern R, Lutterklas M, Egelhaaf M (2000) Neural representation of optic flow experienced by unilaterally blinded flies on their mean walking trajectories. J Comp Physiol A 186:467-479

Kern R, Lutterklas M, Petereit C, Lindemann JP, Egelhaaf M (2001) Neuronal processing of behaviourally generated optic flow: experiments and model simulations. Network-Comp Neural 12:351-369

Kern R, van Hateren JH, Egelhaaf M (2006) Representation of behaviourally relevant information by blowfly motion-sensitive visual interneurons requires precise compensatory head movements. J Exp Biol 209:1251-1260

Kern R, van Hateren JH, Michaelis C, Lindemann JP, Egelhaaf M (2005) Function of a fly motion-sensitive neuron matches eye movements during free flight. PLOS Biol 3:1130-1138

Koenderink JJ, van Doorn AJ (1987) Facts on optic flow. Biol Cybern $56: 247-254$

Kral K, Vernik M, Devetak D (2000) The visually controlled preycapture behaviour of the European Mantisped Mantispa stryriaca. J Exp Biol 203:2117-2123

Krapp HG (2000) Neuronal matched filters for optic flow processing in flying insects. In: Lappe M (ed) Neuronal processing of optic flow. Academic Press, New York, pp 93-120

Krapp HG, Hengstenberg R, Egelhaaf M (2001) Binocular contributions to optic flow processing in the fly visual system. J Neurophysiol 85:724-734

Lindemann JP, Kern R, Michaelis C, Meyer P, van Hateren JH, Egelhaaf M (2003) FliMax, a novel stimulus device for panoramic and highspeed presentation of behaviourally generated optic flow. Vis Res 43:779-791 
Lindemann JP, Kern R, van Hateren JH, Ritter H, Egelhaaf M (2005) On the computations analysing natural optic flow: quantitative model analysis of the blowfly motion vision pathway. J Neurosci 25:6435-6448

Neumann TR (2004) Biomimetic spherical vision. Ph.D. Thesis, Universität Tübingen

Reiser MB, Dickinson MH (2003) A test bed for insect-inspired robotic control. Phil Trans R Soc Lond A 361:2267-2285

Schenato L, Deng X, Wu WC (2001) Virtual insect flight simulator (VIFS): a software testbed for insect flight. In: IEEE Int Conf Robotics and Automation, pp 3885-3892

Schilstra C, van Hateren JH (1998) Stabilizing gaze in flying blowflies. Nature 395:654-654

Schilstra C, van Hateren JH (1999) Blowfly flight and optic flow. I. Thorax kinematics and flight dynamics. J Exp Biol 202:14811490

Sobel EC (1990) The locust's use of motion parallax to measure distance. J Comp Physiol A 167:579-588

Sobey PJ (1994) Active navigation with a monocular robot. Biol Cybern 71:433-440

Srinivasan MV, Zhang SW, Lehrer M, Collett TS (1996) Honeybee navigation en route to the goal: visual flight control and odometry. J Exp Biol 199:237-244

Srinivasan MV, Zhang SW (2000) Visual navigation in flying insects. Int Rev Neurobiol 44:67-92
Tammero LF, Dickinson MH (2002a) Collision-avoidance and landing responses are mediated by separate pathways in the fruit fly, Drosophila melanogaster. J Exp Biol 205:2785-2798

Tammero LF, Dickinson MH (2002b) The influence of visual landscape on the free flight behavior of the fruit fly Drosophila melanogaster. J Exp Biol 205:327-343

van Hateren JH, Kern R, Schwerdtfeger G, Egelhaaf M (2005) Function and coding in the blowfly $\mathrm{H} 1$ neuron during naturalistic optic flow. J Neurosci 25:4343-4352

van Hateren JH, Schilstra C (1999) Blowfly flight and optic flow. II. Head movements during flight. J Exp Biol 202:1491-1500

Warzecha A-K, Egelhaaf M (1996) Intrinsic properties of biological motion detectors prevent the optomotor control system from getting unstable. Phil Trans Roy Soc Lond, B 351:1579-1591

Warzecha A-K, Egelhaaf M (2000) Response latency of a motionsensitive neruon in the fly visual system: dependence on stimulus parameters and physiological conditions. Vis Res 40:2973-2983

Warzecha A-K, Kretzberg J, Egelhaaf M (1998) Temporal precision of the encoding of motion information by visual interneurons. Curr Biol 8:359-368

Webb B, Harrison RR, Willis MA (2004) Sensorimotor control of navigation in arthropod artifical systems. Arthrop Struct Develop 33:301-329

Zufferey J-C, Floreano D (2006) Fly-inspired visual steering of an ultralight indoor aircraft. IEEE Trans Robot 22:137-146 